Pacific Journal of Mathematic 


\title{
BIFURCATION OF OPERATOR EQUATIONS WITH UNBOUNDED LINEARIZED PART
}

\author{
DAVID Westreich
}

\begin{abstract}
The bifuraction problem for the operator equation $x=\lambda L x+G(\lambda, x)$ is considered, where $L$ is a closed linear operator with characteristic value $\lambda_{0}$, and $G(\lambda, x)$ is a continuous higher order term. If $I-\lambda_{0} L$ is a closed Fredholm operator and either $L$ is self-adjoint and $G$ is a continuously differentiable gradient operator or $\lambda_{0}$ is of odd algebraic multiplicity, then $\lambda_{0}$ is shown to be a bifurcation point.
\end{abstract}

Introduction. Several authors have considered the bifurcation problem for nonlinear operator equations with closed linearized part. J. MacBain [7] considered the case where the nonlinear term is compact and obtained global results, similar to those gotten by P. H. Rabinowitz [8] for compact operator equations. Other results in specialized instances were obtained, among others, by M. G. Grandall and P. H. Rabinowitz [3], M. Reeken [10], and R. Böhme [2] who also considered gradient operator equations.

In this note we extend known local bifurcation results, to nonlinear operator equations with linearized parts closed Fredholm operators and continuous higher order terms, dependent on $\lambda$, and where characteristic value is of odd algebraic multiplicity.

Bifurcation results are also obtained for variational equations, though except for the dependence of the higher order term on $\lambda$ they are not as strong as those of Böhme in [2].

1. Preliminary lemmas and definitions. To solve the bifurcation problem for a large class of nonlinear operators with noncontinuous linearized part we must introduce several preliminary definitions and technical lemmas.

The domain of a closed linear operator $T$ of a Banach space $X \rightarrow X$ will be denoted $D(T)$. If $T$ and $B$ are two closed linear operators of $X \rightarrow X$ by $T B$ we will mean the operator defined by $T(B x)$ for $x \in D(T B)=\{x \mid x \in D(B)$ and $B x \in D(T)\}$. The null space and range of $T$ will be denoted $N(T)$ and $R(T)$ respectively. For convenience we write $D\left(T^{k}\right)=D_{k}(T), N\left(T^{k}\right)=N_{k}(T), R\left(T^{k}\right)=R_{k}(T)$ and $\bigcup_{k=1}^{\infty} N_{k}(T)=N_{\infty}(T)$. The smallest integer $k>0$ such that $N_{k}(T)=N_{k+1}(T)$ is called the ascent of $T$ and is denoted by $\alpha(T)$. If there is no such $k$ we say that $\alpha(T)=\infty$. Similarly the smallest integer $k$ such that $R_{k}(T)=R_{k+1}(T)$ is called the descent of $T$ and 
is denoted by $\delta(T)$ and we say $\delta(T)=\infty$ if there is no such $k$.

For the ascent and descent of an operator one can show

Lemma 1. $\quad N_{n}(T) \subseteq N_{n+1}(T), n=0,1, \ldots$ If $N_{k}(T)=N_{k+1}(T)$ for some k then $N_{k+n}(T)=N_{k}(T) . \quad R_{n+1}(T) \subseteq R_{n}(T), n=0,1, \ldots$. If $R_{k+1}(T)=$ $R_{k}(T)$ then $R_{k+n}(T)=R_{k}(T)$. If $\alpha(T)=p<\infty$ and $\delta(T)<\infty$ then $\alpha(T)=\delta(T), R_{p}(T) \cap N_{p}(T)=\{0\}$ and $D_{p}(T)=N_{p}(T) \oplus\left\{R_{p}(T) \cap D_{p}(T)\right\}$.

Proof. See [12, pp. 271-273].

For any Banach space $X$, we denote its conjugate by $X^{*}$ and the conjugate of a linear operator $T$ by $T^{*}$. A closed linear operator $T$ of a real or complex Banach space $X$ into itself is said to be a Fredholm operator if $\overline{D(T)}=X, R(T)$ is closed and both $N(T)$ and $N\left(T^{*}\right)$ are finite. The index of a Fredholm operator $T$, written $\kappa(T)$, is $\operatorname{dim} N(T)-\operatorname{dim} N\left(T^{*}\right)$.

THEOREM 2. Let $T$ and $B$ be two closed Fredholm operators of $X$ into $X$, then $T B$ is a closed Fredholm operator and $\kappa(T B)=$ $\kappa(T)+\kappa(B)$.

Proof. See [5, p. 103].

TheOREM 3. Let $T$ be a closed Fredholm operator of a real or complex Banach space $X$ into $X, T^{*}$ its conjugate and suppose $\operatorname{dim} N_{\infty}(T)<\infty$ and $\operatorname{dim} N_{\infty}\left(T^{*}\right)<\infty$. Then

(i) $\alpha(T)<\infty$ and $\delta(T)<\infty$,

(ii) $R_{p}(T)$ is closed and $X=N_{p}(T) \oplus R_{p}(T)$ where $p=\alpha(T)$,

(iii) $T$ is a one-one map of $R_{p}(T)$ onto $R_{p}(T)$ with bounded inverse,

(iv) $\operatorname{dim} N_{\infty}(T)=\operatorname{dim} N_{\infty}\left(T^{*}\right)$ and $N_{p}\left(T^{*}\right)=N_{\infty}\left(T^{*}\right)$ and

( v ) $\kappa(T)=0$.

Proof. The proof of $\alpha(T)<\infty$ is immediate. That $R_{p}(T)$ is closed follows from Theorem 2 by an induction. To complete the proof of statements (i) and (ii) we first show $\delta(T)<\infty$. Suppose $\delta(T)$ is not finite. Then $R_{i+1}(T)$ is a proper subset of $R_{i}(T)$ for $i=0,1, \ldots$. For each $i>0$, choose an $x_{i} \in R_{i-1}(T)-R_{i}(T)$ and a $y_{i} \in X^{*}$ such that, $y_{i}\left(x_{i}\right)=1, y_{i}\left(x_{j}\right)=0$ for $j<i$ and $y_{i}\left(R_{i}(T)\right)=0$. Each $y_{i} \in N_{i}\left(T^{*}\right)$ [5, p. 59] and as the $x_{i}$ are linearly independent so are the $y_{i}$. Hence $\operatorname{dim} N_{\infty}\left(T^{*}\right)=\infty$, contradicting our hypothesis and so $\delta(T)<\infty$.

As both the ascent and descent of $T$ are finite, if $p=\alpha(T)$ then $N_{p}(T) \cap R_{p}(T)=\{0\}$. Therefore as $\operatorname{dim} N_{p}(T)<\infty, N_{p}(T) \oplus R_{p}(T)$ 
is closed [5, p. 16]. Suppose $X \neq N_{p}(T) \oplus R_{p}(T)$. Then, since $\operatorname{codim} R_{p}(T)=\operatorname{dim} N_{p}\left(T^{*}\right)<\infty$, there exists a finite dimensional subspace $M$ such that $X=N_{p}(T) \oplus R_{p}(T) \oplus M$, [5, p. 103]. Moreover, as $T^{p}$ is the product of closed Fredholm operators

$$
\overline{D_{p}(T)}=X \text {. }
$$

Thus

$$
\overline{D_{p}(T)}=N_{p}(T) \oplus\left\{R_{p}(T) \cap \overline{D_{p}(T)}\right\} \oplus M .
$$

On the other hand by Lemma 1

$$
D_{p}(T)=N_{p}(T) \oplus\left\{R_{p}(T) \cap D_{p}(T)\right\} .
$$

Clearly

$$
N_{p}(T) \oplus\left\{R_{p}(T) \cap D_{p}(T)\right\} \subseteq N_{p}(T) \oplus\left\{R_{p}(T) \cap \overline{D_{p}(T)}\right\} .
$$

Thus Eqs. (1), (2), (3) and (4) imply $M=\{0\}$ and we have $X=N_{p}(T) \oplus$ $R_{p}(T)$. Statement (iii) is now immediate from statement (ii), Lemma 1 and the bounded inverse theorem [12, p. 179].

Lastly, we show statement (iv). From statement (ii) it follows that $X^{*}=R_{p}(T)^{\perp} \oplus N_{p}(T)^{\perp}$ [5, p. 100] where for $U \leqq X, U^{\perp}=\{y \in$ $\left.X^{*} \mid y(x)=0, x \in U\right\} . \quad$ The $\operatorname{dim} R_{p}(T)^{\perp}=\operatorname{dim} N_{p}(T), R_{p}(T)^{\perp}=N_{p}\left(T^{*}\right)$ [5, p. 51] and $N_{p}(T)^{\perp}=R_{p}\left(T^{*}\right)$ [5 p. 95]. Thus $X^{*}=N_{p}\left(T^{*}\right) \oplus R_{p}\left(T^{*}\right)$ and so $N_{p}\left(T^{*}\right)=N_{\infty}\left(T^{*}\right)$, which in turn implies

$$
\operatorname{dim} N_{\infty}(T)=\operatorname{dim} N_{p}(T)=\operatorname{dim} N_{p}\left(T^{*}\right)=\operatorname{dim} N_{\infty}\left(T^{*}\right) .
$$

To show statement (v) we note that statement(iv) implies $\kappa\left(T^{p}\right)=0$. But by an induction it follows from Theorem 2 that $\kappa\left(T^{p}\right)=p \kappa(T)$. Hence $\kappa(T)=0$.

2. Odd multiplicity results. Let $X$ be a real Banach space and suppose $L$ is a densely defined closed linear map of $X$ into $X$ with a real characteristic value $\lambda_{0}$, that is there exists a nonzero $x_{0} \in X$ such that $\lambda_{0} L x_{0}=x_{0}$. The algebraic multiplicity of $\lambda_{0}$ is defined to be $\operatorname{dim} N_{\infty}\left(I-\lambda_{0} L\right)$. Suppose further $G(\lambda, x)$ is a continuous map of a neighborhood of $\left(\lambda_{0}, 0\right) \in \boldsymbol{R} \times X$ into $X$ satisfying

$$
\left\|G\left(\lambda, x_{1}\right)-G\left(\lambda, x_{2}\right)\right\|=h\left(x_{1}, x_{2}\right)\left\|x_{1}-x_{2}\right\|
$$

for $(\lambda, x)$ near $\left(\lambda_{0}, 0\right)$ and where $h(a, b)$ is a function independent of $\lambda$ tending to zero as both $a$ and $b$ tend to zero. We shall be concerned with finding nontrivial solutions, that is points $(\lambda, x) \in \boldsymbol{R} \times X, x \neq 0$, satisfying the equation

$$
x=\lambda L x+G(\lambda, x) .
$$


The closure of the set of nontrivial solutions of (6) will be denoted by $S$. We will call $\lambda_{0}$ a bifurcation point of Eq. (6) if every neighborhood of $\left(\lambda_{0}, 0\right)$ contains a nontrivial solution of (6). Using a topological degree argument, P. H. Rabinowitz [8] proved the following bifurcation result:

THEOREM 4. If $L$ is completely continuous, $\Omega$ is a bounded open set in $\boldsymbol{R} \times X$ containing $\left(\lambda_{0}, 0\right), G(\lambda, x)$ is completely continuous on $\bar{\Omega}$ and $\lambda_{0}$ is a characteristic value of odd algebraic multiplicity, then there is a maximal closed connected subset $C$ of $S$ such that $C \subseteq \bar{\Omega}$, $\left(\lambda_{0}, 0\right) \in C$ and $C$ either meets the boundary of $\Omega$ or meets $(\hat{\lambda}, 0)$, where $\hat{\lambda}$ is another characteristic value of $L$.

By methods somewhat similar to those of [7] we can obtain a partial extension of Theorem 4 to those instance where Eq. (6) is not completely continuous and indeed $L$ is not even bounded.

Theorem 5. Let $L$ and $G$ be as described above. Suppose $I-\lambda_{0} L$ is a closed Fredholm operator and $\lambda_{0}$ is a characteristic value of odd algebraic multiplicity of $L$ and a characteristic value of finite algebraic multiplicity of $L^{*}$. Then there exists a maximal closed connected subset of $S$ meeting $\left(\lambda_{0}, 0\right)$ and $\lambda_{0}$ is a bifurcation point.

Proof. By Theorem 3, $X=N \oplus R$ where $N=N_{\infty}\left(I-\lambda_{0} L\right)$ and $R=R_{\infty}\left(I-\lambda_{0} L\right)$. Thus $x \in X$ can be uniquely expressed as $x=u+v$ where $u \in N$ and $v \in R$. Moreover for all $\lambda \in R, I-\lambda L: N \rightarrow N$, $R \rightarrow R$ and $G(\lambda, x)=G_{N}(\lambda, x)+G_{R}(\lambda, x)$ where $G_{N}(\lambda, x) \in N$ and $G_{R}(\lambda, x) \in R$. Thus our problem is equivalent to that of finding solutions $(\lambda, u, v) \in \boldsymbol{R} \times N \times R$ of the system of equations

$$
\begin{aligned}
& u-\lambda L u=G_{N}(\lambda, u+v) \\
& v-\lambda L v=G_{R}(\lambda, u+v) .
\end{aligned}
$$

Since $I-\lambda L$ has a bounded inverse on $R$ for $\lambda$ near $\lambda_{0}$ and $(I-\lambda L)^{-1}$ is continuous in $\lambda$ for all $1 / \lambda$ in the resolvent of $L$ (as a mapping of $R \rightarrow R)$ [12, p. 257], $(\lambda, u, v)$ is a solution of (6a), (6b) if and only if $(\lambda, u, v)$ is a solution of the system

$$
\begin{aligned}
& u=\lambda L u+G_{N}(\lambda, u+v) \\
& v=(I-\lambda L)^{-1} G_{R}(\lambda, u+v) .
\end{aligned}
$$

An application of the contraction mapping principle [4, p. 260] to Eq. (7b) shows the existence of a uniquely determined continuous 
function, $v(\lambda, u)=v$ such that $v(\lambda, u)=(I-\lambda L)^{-1} G_{R}(\lambda, u+v(\lambda, u))$ for all $(\lambda, u)$ in a neighborhood of $\left(\lambda_{0}, 0\right)$. Consequently it suffices to find solutions in $R \times N$ of the equation

$$
u=\lambda L u+G_{N}(\lambda, u+v(\lambda, u)) .
$$

By continuity Eq. (8) satisfies the hypothesis of Theorem 4 near $\left(\lambda_{0}, 0\right)$ thus there exists a closed connected subset $C^{\prime}$ of $S$ meeting $\left(\lambda_{0}, 0\right)$ and $\lambda_{0}$ is a bifurcation point. As the union of connected sets containing a common point is connected an application of Zorn's lemma [6, p. 62] will show that $S$ contains a unique maximal closed connected subset $C$ meeting $\left(\lambda_{0}, 0\right)$. Thus $C^{\prime} \subseteq C$ and our theorem is proven.

REMARK. Rabinowitz [9, p. 17] has proven a result similar to Theorem 5 for $L$ bounded.

3. Gradient operators. Let $X$ be a real Hilbert space and suppose $L$ is a densely defined closed self-adjoint linear operator of $X$ into $X$ with a characteristic value $\lambda_{0}$. Suppose further $G(\lambda, x)$ is a twice continuously differentiable map of a neighborhood of $\left(\lambda_{0}, 0\right) \in$ $\boldsymbol{R} \times X$ into $X$ such that for fixed $\lambda, G(\lambda, x)$ is a gradient map [13, p. 54] and $G(\lambda, 0) \equiv 0$ and $G_{x}(\lambda, 0) \equiv 0$ for all $\lambda$ near $\lambda_{0}$. We shall be concerned with solving the bifurcation problem for the equation

$$
x=\lambda L x+G(\lambda, x) .
$$

For $L$ bounded, M. S. Berger [1] has shown

THEOREM 6. If $L$ is bounded and $I-\lambda_{0} L$ is a Fredholm operator then $\lambda_{0}$ is a bifurcation point of Eq. (9).

The same result may be obtained for $L$ unbounded but closed.

THEOREM 7. If $I-\lambda_{0} L$ is a Fredholm operator then $\lambda_{0}$ is a bifurcation point of Eq. (9).

Proof. As in the proof of Theorem 5 it suffices to solve the bifurcation problem for the system of equations

$$
\begin{aligned}
& u=\lambda L u+G_{N}(\lambda, u+v) \\
& v=(I-\lambda L)^{-1} G_{R}(\lambda, u+v)
\end{aligned}
$$

for $(\lambda, u, v) \in R \times N \times R, G_{N} \in N$ and $G_{R} \in R$, where $N=N\left(I-\lambda_{0} L\right)$ and $R=R\left(I-\lambda_{0} L\right)$. By the implicit function theorem [4, p. 265] there exists a uniquely determined, twice continuously differentiable 
function $v(\lambda, u)$ such that

$$
v(\lambda, u)=(I-\lambda L)^{-1} G_{R}(\lambda, u+v(\lambda, u))
$$

for all $(\lambda, u)$ near $\left(\lambda_{0}, 0\right)$. Thus our problem is reduced to solving the operator equation

$$
u=\lambda L u+G_{N}(\lambda, u+v(\lambda, u))
$$

for $(\lambda, u) \in \boldsymbol{R} \times N$. Moreover, if for fixed $\lambda, \mathscr{G}(\lambda, x)$ is the potential of $G(\lambda, x)$ (that is $\mathscr{G}_{x}(\lambda, x)=G(\lambda, x)$ ) then arguing as in the proof of Theorem 1 in [14] one can readily verify that for fixed $\lambda$

$$
\frac{1}{2}\langle(I-\lambda L)(u+v(\lambda, u)), u+v(\lambda, u)\rangle-\mathscr{C}(\lambda, u+v(\lambda, u))
$$

is a potential for $(I-\lambda L) u-G_{N}(\lambda, u+v(\lambda, u)) . \quad(\langle$,$\rangle is the inner$ product on $X$.)

Therefore Eq. (10) is a gradient operator equation. Hence, as (10) satisfies the hypothesis of Theorem $6, \lambda_{0}$ must be a bifurcation point of Eq. (9).

4. Remarks and two counterexamples. By Theorem 3 we could have replaced the hypothesis on the multiplicity of $\lambda_{0}$ of Theorem 5 by the equivalent hypothesis $\lambda_{0}$ is a characteristic value of the same odd algebraic multiplicity of both $L$ and $L^{*}$. Moreover we could have alternately assumed $\lambda_{0}$ a characteristic value of odd algebraic multiplicity and $I-\lambda_{0} L$ is a Fredholm operator of index zero since we can show

THEOREM 8. Suppose $T$ is a closed linear Fredholm operator of $X$ into $X$. If $\operatorname{dim} N_{\infty}(T)<\infty$ and $\kappa(T)=0$ then $\operatorname{dim} N_{\infty}\left(T^{*}\right)=\operatorname{dim} N_{\infty}(T)$.

Proof. Let $p=\alpha(T)$. Then by Theorem $2, \kappa\left(T^{p}\right)=p \kappa(T)=0$. As in the proof of Theorem $3, N_{p}(T) \oplus R_{p}(T) \oplus M=X$. If $M \neq\{0\}$ then $\operatorname{dim} N_{p}\left(T^{*}\right)>\operatorname{dim} N_{p}(T)$ which is impossible as $\kappa\left(T^{p}\right)=0$. Hence $X=N_{p}(T) \oplus R_{p}(T)$ which implies $X^{*}=N_{p}\left(T^{*}\right) \oplus R_{p}\left(T^{*}\right)$ and so $\operatorname{dim} N_{\infty}\left(T^{*}\right)=\operatorname{dim} N_{p}\left(T^{*}\right)=\operatorname{dim} N_{p}(T)$.

We give an example to show that if $\lambda_{0}$ is a characteristic value of odd algebraic multiplicity of $L$ but $\kappa\left(I-\lambda_{0} L\right)<0$ then $\lambda_{0}$ may fail to be a bifurcation point.

Let $H=\ell_{2} \times \boldsymbol{R}$ and let $S$ be the shift operator on $\ell_{2}$, that is $S:\left(a_{1}, a_{2}, \cdots\right) \rightarrow\left(0, a_{1}, a_{2}, \cdots\right)$ and consider the equations

$$
\begin{aligned}
& x=\lambda\left[(S+I) x+\left(y^{2}, 0, \cdots\right)\right] \\
& y=\lambda y
\end{aligned}
$$


for $(\lambda, x, y) \in \boldsymbol{R} \times H=\boldsymbol{R} \times\left(\ell_{2} \times \boldsymbol{R}\right) . \quad \lambda_{0}=1$ is a characteristic value of linear part of odd multiplicity and as

$$
\left(\begin{array}{cc}
I-\lambda_{0}(S+I) & 0 \\
0 & I-\lambda_{0} I
\end{array}\right)=-\left(\begin{array}{ll}
S & 0 \\
0 & 0
\end{array}\right)=L
$$

we see $\kappa(L)<0$. Moreover a simple examination of cases for $y=0$ or $y \neq 0$ shows that $\lambda_{0}=1$ is not a bifurcation point of the system.

Lastly we show that if $\lambda_{0}$ is a characteristic value of odd geometric multiplicity (that is $\operatorname{dim} N\left(I-\lambda_{0} L\right)$ is odd) but $\kappa\left(I-\lambda_{0} L\right)>0$ then $\lambda_{0}$ may fail to be a bifurcation point.

Let $x, y \in \ell_{2}$ and consider the system of equations

$$
\begin{aligned}
& x=\lambda\left(S^{2}+I\right) x+\lambda\left(\|x\|^{2}+\|y\|_{1}^{2}, 0, \cdots\right) \\
& y=\lambda\left(S^{*^{3}}+I\right) y
\end{aligned}
$$

where $S^{*}$ is the adjoint of $S$, that is the left shift operator $S^{*}:\left(y_{1}, y_{2}, \cdots\right) \rightarrow\left(y_{2}, y_{3}, \cdots\right)$.

If $\lambda_{0}=1$ then the dimension of the null space of the linearized part of the system, $I-\lambda_{0} L$, is equal to 3 and $\operatorname{dim} N\left(I-\lambda_{0} L^{*}\right)=2$. Thus $\kappa\left(I-\lambda_{0} L\right)=3-2>0$. Moreover for $\lambda$ near $\lambda_{0}$ the system has no solutions other than trivial ones. Indeed, suppose $(\lambda, x, y)$ is a nontrivial solution and $x=\left(x_{1}, x_{2}, \cdots\right)$. Then we have the equality

$$
(I-\lambda)\left(x_{1}, x_{2}, \cdots\right)=\lambda\left(0,0, x_{1}, x_{2}, \cdots\right)+\lambda\left(\|x\|^{2}+\|y\|^{2}, 0, \cdots\right) .
$$

Thus $x_{1}=\lambda(1-\lambda)^{-1}\left(\|x\|^{2}+\|y\|^{2}\right)$ and for $n=1, \cdots, x_{2 n}=0$ and $x_{2 n+1}=\lambda(1-\lambda)^{-1} x_{2 n-1}$. Therefore

$$
x=\left(\|x\|^{2}+\|y\|^{2}\right)\left(\lambda(1-\lambda)^{-1}, 0, \lambda^{2}(1-\lambda)^{-2}, \cdots\right) .
$$

However as $\lambda^{n}(1-\lambda)^{-n} \nrightarrow 0$ as $n \rightarrow \infty$ for $1 / 2 \leqq \lambda, x \notin \ell_{2}$. Hence the system does not have any nontrivial solutions for $\lambda$ near $\lambda_{0}=1$.

\section{REFERENCES}

1. M. S. Berger, Bifurcation theory and the type number of Marston Morse, Proc. National Academy of Sci., 69 (1972), 1737-1738.

2. R. Böhme, Die Lösung der Verzweigungsgleichungen für nichtlineare Eigenwert probleme, Math. Zeit., 127 (1972), 105-126.

3. M. G. Crandall and P. H. Rabinowitz, Bifurcation from simple eigenvalues, J. Functional Analysis, 18 (1971), 321-340.

4. J. Dieudonne, Foundation of Modern Analysis, Academic Press, New York 1960.

5. S. Goldberg, Unbounded Linear Operators, McGraw-Hill, New York, 1966.

6. P. Halmos, Naive Set Theory, D. van Nostrand Co., Inc. Princeton, 1966.

7. J. MacBain, Global bifurcation theorems for noncompact operators, Bull. Amer. Math. Soc., 80 (1974), 1005-1009.

8. P. H. Rabinowitz, Some global results for nonlinear eigenvalue problems, J. Functional Analysis, 7 (1971), 487-513. 
9. — A global theorem for nonlinear eigenvalue problems and applications, Contributions to Nonlinear Functional Analysis. edited by E. H. Zarantonello, Academic Press, N.Y., 1971.

10. M. Reeken, General theorem on bifurcation and its application to the Hartree equation for the helium atom, J. Math. Phys., 11 (1970), 2505-2512.

11. F. Riesz and B. Sz.-Nagy, Functional Analysis, Fredrick Ungar Publishing Co., New York, 1955.

12. A. E. Taylor, Introduction to Functional Analysis, John Wiley \& Sons Inc., New York, 1958.

13. M. M. Vainberg, Variational Methods for the Study of Nonlinear Operators, Holden-Day Inc., San-Francisco, 1964.

14. D. Westreich, Periodic solutions of second order Lagrangian systems, Duke Math. J., 41 (1974), 405-411.

Received April 12, 1974 and in revised form February 21, 1975.

Ben-Gurion University of THE NegeV

Beer-Sheva, Israel 


\section{PACIFIC JOURNAL OF MATHEMATICS}

\section{EDITORS}

RICHARD ARENS (Managing Editor)

University of California

Los Angeles, California 90024

\section{J. DugundJI}

Department of Mathematics University of Southern California Los Angeles, California 90007

D. Gilbarg and J. Milgram

Stanford University

Stanford, California 94305
University of Washington Seattle, Washington 98105

\section{ASSOCIATE EDITORS}
E. F. BECKENBACH
B. H. NeumanN
F. WolF
K. YoShIDA

\section{SUPPORTING INSTITUTIONS}

\author{
UNIVERSITY OF SOUTHERN CALIFORNIA \\ STANFORD UNIVERSITY \\ UNIVERSITY OF TOKYO \\ UNIVERSITY OF UTAH \\ WASHINGTON STATE UNIVERSITY \\ UNIVERSITY OF WASHINGTON \\ $\stackrel{*}{*} \stackrel{*}{*} \stackrel{*}{ }{ }^{*}$ AMERICAN MATHEMATICAL SOCIETY
}

The Supporting Institutions listed above contribute to the cost of publication of this Journal, but they are not owners or publishers and have no responsibility for its content or policies.

Mathematical papers intended for publication in the Pacific Journal of Mathematics should be in typed form or offset-reproduced, (not dittoed), double spaced with large margins. Underline Greek letters in red, German in green, and script in blue. The first paragraph or two must be capable of being used separately as a synopsis of the entire paper. Items of the bibliography should not be cited there unless absolutely necessary, in which case they must be identified by author and Journal, rather than by item number. Manuscripts, in triplicate, may be sent to any one of the editors. Please classify according to the scheme of Math. Reviews, Index to Vol. 39. All other communications should be addressed to the managing editor, or Elaine Barth, University of California, Los Angeles, California, 90024.

The Pacific Journal of Mathematics expects the author's institution to pay page charges, and reserves the right to delay publication for nonpayment of charges in case of financial emergency.

100 reprints are provided free for each article, only if page charges have been substantially paid. Additional copies may be obtained at cost in multiples of 50 .

The Pacific Journal of Mathematics is issued monthly as of January 1966. Regular subscription rate: $\$ 72.00$ a year (6 Vols., 12 issues). Special rate: $\$ 36.00$ a year to individual members of supporting institutions.

Subscriptions, orders for back numbers, and changes of address should be sent to Pacific Journal of Mathematics, 103 Highland Boulevard, Berkeley, California, 94708.

\section{PUBLISHED BY PACIFIC JOURNAL OF MATHEMATICS, A NON-PROFIT CORPORATION}

Printed at Kokusai Bunken Insatsusha (International Academic Printing Co., Ltd.), 270, 3-chome Totsuka-cho, Shinjuku-ku, Tokyo 160, Japan.

\section{Copyright (C) 1975 by Pacific Journal of Mathematics} Manufactured and first issued in Japan 


\section{Pacific Journal of Mathematics}

\section{Vol. 57, No. $2 \quad$ February, 1975}

Norman Larrabee Alling, On Cauchy's theorem for real algebraic curves with boundary .......

Daniel D. Anderson, A remark on the lattice of ideals of a Prüfer domain ..................

Dennis Neal Barr and Peter D. Miletta, A necessary and sufficient condition for uniqueness of

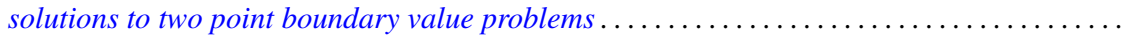

Ladislav Beran, On solvability of generalized orthomodular lattices . . . . . . . . . . ........

L. Carlitz, A three-term relation for some sums related to Dedekind sums . . . . . . . . . .....

Arthur Herbert Copeland, Jr. and Albert Oscar Shar, Images and pre-images of localization

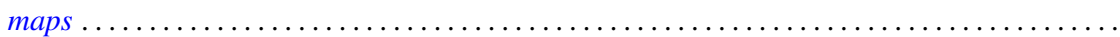

G. G. Dandapat, John L. Hunsucker and Carl Pomerance, Some new results on odd perfect

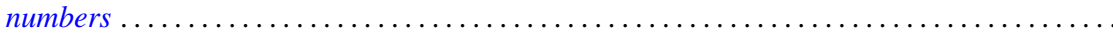

M. Edelstein and L. Keener, Characterizations of infinite-dimensional and nonreflexive

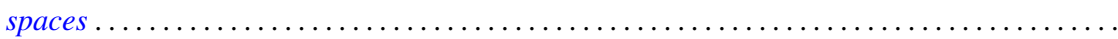

Francis James Flanigan, On Levi factors of derivation algebras and the radical embedding

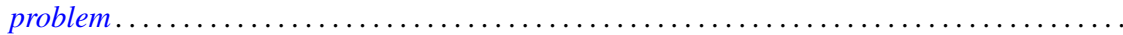

Harvey Friedman, Provable equality in primitive recursive arithmetic with and without

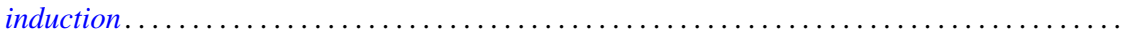

Joseph Braucher Fugate and Lee K. Mohler, The fixed point property for tree-like continua with

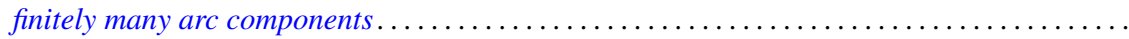

John Norman Ginsburg and Victor Harold Saks, Some applications of ultrafilters in

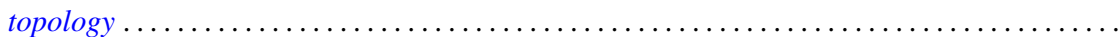

Arjun K. Gupta, Generalisation of a "square" functional equation .....................

Thomas Lee Hayden and Frank Jones Massey, Nonlinear holomorphic semigroups ..........

V. Kannan and Thekkedath Thrivikraman, Lattices of Hausdorff compactifications of a locally

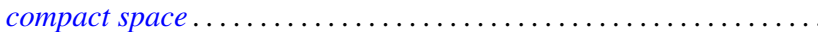

J. E. Kerlin and Wilfred Dennis Pepe, Norm decreasing homomorphisms between group

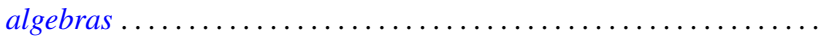

Young K. Kwon, Behavior of $\Phi$-bounded harmonic functions at the Wiener boundary ...

Richard Arthur Levaro, Projective quasi-coherent sheaves of modules .

Chung Lin, Rearranging Fourier transforms on groups...........................

David Lowell Lovelady, An asymptotic analysis of an odd order linear differential equation . . 4475

Jerry Malzan, On groups with a single involution .......................... 481

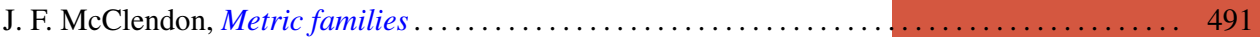

Carl Pomerance, On multiply perfect numbers with a special property .

Mohan S. Putcha and Adil Mohamed Yaqub, Polynomial constraints for finiteness of semisimple rings. .

Calvin R. Putnam, Hyponormal contractions and strong power convergence . . . . . . . . . 531

Douglas Conner Ravenel, Multiplicative operations in $\mathrm{BP} * \mathrm{BP} \ldots \ldots \ldots \ldots \ldots \ldots \ldots \ldots \ldots .539$

Judith Roitman, Attaining the spread at cardinals which are not strong limits . . . . . . . . . 545

Kazuyuki Saitô, Groups of *-automorphisms and invariant maps of von Neumann algebras . . . 553

Brian Kirkwood Schmidt, Homotopy invariance of contravariant functors acting on smooth

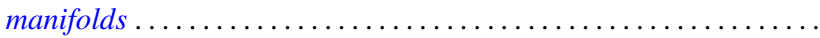

Kenneth Barry Stolarsky, The sum of the distances to $N$ points on a sphere.

Mark Lawrence Teply, Semiprime rings with the singular splitting property.

J. Pelham Thomas, Maximal connected Hausdorff spaces..............

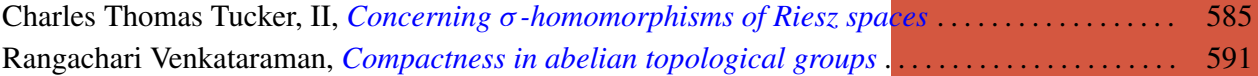

William Charles Waterhouse, Basically bounded functors and flat sheaves . . . . . . . . . . . 597

David Westreich, Bifurcation of operator equations with unbounded linearized part ......... 611

William Robin Zame, Extendibility, boundedness and sequential convergence in spaces of 